\title{
Static and dynamic alterations in the amplitude of low- frequency fluctuation in patients with amyotrophic lateral sclerosis
}

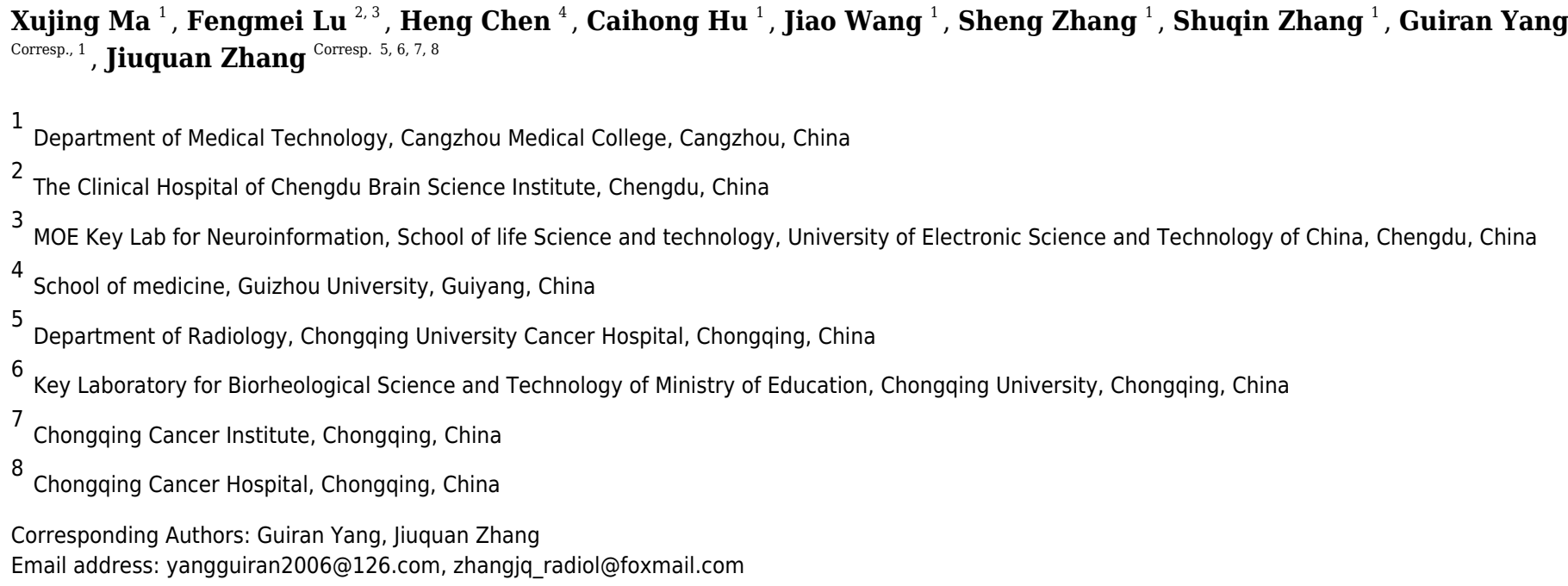

Background: Static changes in local brain activity in patients suffering from amyotrophic lateral sclerosis (ALS) have been studied. However, the dynamic characteristics of local brain activity are poorly understood. Whether dynamic alterations could differentiate patients with ALS from healthy controls (HCs) remains unclear. Methods: A total of 54 patients with ALS (mean age $=48.71$ years, male/female $=36 / 18$ ) and 54 (mean age $=$ 48.30 years, male/female $=36 / 18$ ) HCs underwent magnetic resonance imaging scans. To depict static alterations in cortical activity, amplitude of low-frequency fluctuations (ALFF) which measures the total power of regional activity was computed. Dynamic ALFF (d-ALFF) from all subjects was calculated using a sliding-window approach. Statistical differences in ALFF and d-ALFF between both groups were used as features to explore whether they could differentiate ALS from HC through support vector machine method. Results: In contrast with HCs, patients with ALS displayed increased ALFF in the right inferior temporal gyrus and bilateral frontal gyrus and decreased ALFF in the left middle occipital gyrus and left precentral gyrus. Furthermore, patients with ALS demonstrated lower d-ALFF in widespread regions, including the right lingual gyrus, left superior temporal gyrus, bilateral precentral gyrus, and left paracentral lobule by comparison with HCs. Furthermore, the ALFF in the left superior orbitofrontal gyrus had a tendency of correlation with ALSFRS-R score and disease progression rate. The classification performance in distinguishing ALS was higher with both features of ALFF and d-ALFF than that with a single approach. 
Conclusions: Decreased dynamic brain activity in the precentral gyrus, paracentral gyrus, lingual gyrus, and temporal regions was found in ALS group. The combined ALFF and dALFF could distinguish ALS from HCs with a higher accuracy than ALFF and d-ALFF alone. These findings may provide important evidence for understanding the neuropathology underlying ALS. 


\section{Static and dynamic alterations in the amplitude of low-}

2 frequency fluctuation in patients with amyotrophic

\section{3 lateral sclerosis}

4

5

6 Xujing $\mathrm{Ma}^{1}$, Fengmei $\mathrm{Lu}^{2,3}$, Heng $\mathrm{Chen}^{4}$, Caihong $\mathrm{Hu}^{1}$, Jiao Wang ${ }^{1}$, Sheng Zhang ${ }^{1}$, Shuqin

7 Zhang ${ }^{1}$, Guiran Yang ${ }^{*}$, Jiuquan Zhang ${ }^{5,6,7,8 *}$

8

$9{ }^{1}$ Department of Medical Technology, Cangzhou Medical College, Cangzhou, China. 10

${ }^{2}$ The Clinical Hospital of Chengdu Brain Science Institute, Chengdu, China.

${ }^{3}$ MOE Key Lab for Neuroinformation, School of life Science and technology, University of Electronic Science and Technology of China, Chengdu, China.

${ }^{4}$ School of medicine, Guizhou University, Guiyang, China.

${ }^{5}$ Department of Radiology, Chongqing University Cancer Hospital, Chongqing, China

${ }^{6}$ Key Laboratory for Biorheological Science and Technology of Ministry of Education, Chongqing University, Chongqing, China.

${ }^{7}$ Chongqing Cancer Institute, Chongqing, China.

${ }^{8}$ Chongqing Cancer Hospital, Chongqing, China.

Corresponding Author:

Jiuquan Zhang

Chongqing 400030, PR China.

Email address: zhangjq_radiol@foxmail.com.

And

Guiran Yang

Cangzhou 061001, PR China.

E-mail: yangguiran2006@126.com. 
30

31

32

33

34

35

36

37

38

39

40

41

42

43

44

45

46

47

48

49

50

51

52

53

54

55

56

57

58

59

60

\section{Abstract}

Background: Static changes in local brain activity in patients suffering from amyotrophic lateral sclerosis (ALS) have been studied. However, the dynamic characteristics of local brain activity are poorly understood. Whether dynamic alterations could differentiate patients with ALS from healthy controls (HCs) remains unclear.

Methods: A total of 54 patients with ALS (mean age $=48.71$ years, male/female $=36 / 18$ ) and 54 (mean age $=48.30$ years, male/female $=36 / 18)$ HCs underwent magnetic resonance imaging scans. To depict static alterations in cortical activity, amplitude of low-frequency fluctuations (ALFF) which measures the total power of regional activity was computed. Dynamic ALFF (dALFF) from all subjects was calculated using a sliding-window approach. Statistical differences in ALFF and d-ALFF between both groups were used as features to explore whether they could differentiate ALS from HC through support vector machine method.

Results: In contrast with HCs, patients with ALS displayed increased ALFF in the right inferior temporal gyrus and bilateral frontal gyrus and decreased ALFF in the left middle occipital gyrus and left precentral gyrus. Furthermore, patients with ALS demonstrated lower d-ALFF in widespread regions, including the right lingual gyrus, left superior temporal gyrus, bilateral precentral gyrus, and left paracentral lobule by comparison with HCs. Furthermore, the ALFF in the left superior orbitofrontal gyrus had a tendency of correlation with ALSFRS-R score and disease progression rate. The classification performance in distinguishing ALS was higher with both features of ALFF and d-ALFF than that with a single approach.

Conclusions: Decreased dynamic brain activity in the precentral gyrus, paracentral gyrus, lingual gyrus, and temporal regions was found in ALS group. The combined ALFF and d-ALFF could distinguish ALS from HCs with a higher accuracy than ALFF and d-ALFF alone. These findings may provide important evidence for understanding the neuropathology underlying ALS.

\section{Introduction}

Amyotrophic lateral sclerosis (ALS) is a devastating disease which involves dysfunctions in movement and cognition (Hardiman et al. 2017; van Es et al. 2017). Patients with ALS usually died within 3-5 years after symptoms appear (Roth \& Shacka 2009). At present, the therapeutic options for ALS are limited. Nevertheless, increasing lines of evidence demonstrate that early diagnosis is important for selecting available pharmacologic therapy and that appropriate palliative care has an active influence on patients' living quality and survival (Bourke et al. 2006; 
61 Volanti et al. 2011). Now the diagnosis of ALS is still clinical, and a pronounced delay exists

62 between the onset of symptoms and diagnosis, possibly beyond the therapeutic window (Turner

63 et al. 2009). Timely and accurate diagnosis of ALS is urgently needed to date, and imaging

64 biomarkers should be developed.

65 Recent studies combining functional and structural data depicted that functional alterations

66 at resting state may precede structural changes in patients with cognitive impairments (Kawagoe

67 et al. 2019; Sun et al. 2016) and ALS (Abidi et al. 2020; Chipika et al. 2019). Neuroimaging

68 approaches provide convenience for studying local brain activities and may facilitate expanding

69 our understanding of early diagnosis of ALS (Huynh et al. 2016; Verstraete \& Foerster 2015).

70 Resting-state functional magnetic resonance imaging (rs-fMRI) is an ideal instrument used to

71 probe into cortical activities based on blood oxygenation level-dependent signals without

72 performing variable tasks (Biswal et al. 1995). As an effective index to measure local brain

73 activity, amplitude of low frequency fluctuation (ALFF) (Guo et al. 2012; Liu et al. 2013; Yu-

74 Feng et al. 2007) has been extensively employed in ALS research. Using this approach, scholars

75 have discovered that patients with ALS showed aberrant activation in the precentral gyrus,

76 frontal gyrus, and occipital regions; this finding suggests that ALS is a disease involving many

77 system with brain impairment spreading beyond the motor cortex (Bueno et al. 2019; Ma et al.

78 2016; Shen et al. 2018). In addition, the increased ALFF in the frontal lobe could be a candidate

79 biomarker in ALS (Luo et al. 2012). However, these studies are on the strength of the hypothesis

80 that the signal of rs-fMRI is static during scanning, ignoring the dynamic behavior of activities of

81 people's brains (Allen et al. 2014; Fu et al. 2017; Liu et al. 2017).

82 Dynamic amplitude of low-frequency fluctuation (d-ALFF), an indicator of the variance of

83 ALFF, is an effective tool to explore brain dynamics in healthy people (Liao et al. 2019; Zou et

84 al. 2009) and patients with neuropsychiatric disorders, including schizophrenia (Yang et al.

85 2019), generalized anxiety disorder (GAD) (Cui et al. 2019), and Parkinson's disease (Zhang et

86 al. 2019). In addition, $\mathrm{Li}$ et al. (Li et al. 2018a) discovered that in contrast to static ALFF

87 abnormalities, d-ALFF abnormalities could predict the severity of suicidal ideation in major

88 depressive disorders. d-ALFF may contribute more than ALFF in differentiating between

89 patients diagnosed with GAD and normal controls (Cui et al. 2019). However, as far as we know,

90 the dynamic signatures of ALFF have been rarely elucidated in ALS; furthermore, the

91 performance of d-ALFF compared with ALFF in recognizing ALS patients from healthy controls 
92 (HCs) at an individual level remains poorly documented.

93 Motivated by previous studies, we utilized d-ALFF to detect changes of dynamic patterns of 94 brain activity in ALS. We assumed that patients with ALS would exhibit altered d-ALFF 95 patterns contrast to $\mathrm{HCs}$, and that such changes could be used as features to distinguish them. We 96 also hypothesized that the classification performance with altered d-ALFF as features would be 97 comparable with that using altered static ALFF.

\section{Materials \& Methods}

\section{Subjects}

100 In western countries, the rates of ALS is probably between 1 and 3 per 100000 per year per 101 person-years (Robberecht \& Philips 2013), while the exact prevalence in China remains unclear (Chen et al. 2015). ALS is more widespread in men than in women in different countries (Chen et al. 2015; Oskarsson et al. 2018). Patients were employed from January 1, 2009 to December 31, 2013 in this work. Fifty-four patients diagnosed with ALS and $54 \mathrm{HCs}$ matched in gender and age were enrolled from Southwest Hospital. The inclusion criteria were as follows: patients can lie down flat in the scanner for at least 40 minutes and receive none therapeutic interventions before participating in this study; and patients with ALS diagnosed on the basis of the revised El Escorial criteria of the World Federation of Neurology (Brooks et al. 2000). The exclusion criteria were as follows: patients diagnosed with frontotemporal dementia or other mental and neurological disorders; patients with major systemic diseases; patients with family trait of motor neuron diseases and other neurodegenerative disorders; and patients with cognitive impairment.

112 The clinical status based on ALS Functional Rating Scale-Revised (ALSFRS-R) was obtained 113 for each patient. Disease duration was computed from symptom onset to examination date. By 114 using the equation: (48-ALSFRS-R score)/Disease duration (Ellis et al. 1999), rate of disease 115 progression was achieved. Demographic and clinical information of subjects are displayed in 116 Table 1.

117 The measurements of the Edinburgh Handedness Inventory indicated that all the subjects 118 were right-handed. The medical research ethics committee of Southwest Hospital (the First 119 Affiliated Hospital of the Third Military Medical University of the Chinese People's Liberation 120 Army) authorized this study to proceed. Informed consent from each participant was collected. 
122 Data were collected as described in our former research (Ma et al. 2015). The following 123 parameters were used in collecting functional data: echo time (TE) $=30 \mathrm{~ms}$, repetition time (TR)

$124=2000 \mathrm{~ms}$, flip angle $(\mathrm{FA})=90^{\circ}, 36$ slices, $1 \mathrm{~mm}$ gap, field of view $(\mathrm{FOV})=192 \mathrm{~mm} \times 192$ $125 \mathrm{~mm}$, thickness $=3 \mathrm{~mm}$, matrix size $=64 \times 64$ and voxel size $=3 \mathrm{~mm} \times 3 \mathrm{~mm} \times 3 \mathrm{~mm}$. Two 126 hundred and forty volumes were collected for each subject. T1-weighted structural data was 127 gathered using the following settings: $\mathrm{TE}=2.52 \mathrm{~ms}, \mathrm{TR}=1900 \mathrm{~ms}, \mathrm{FA}=9^{\circ}$, slice thickness $=1$ $128 \mathrm{~mm}, 176$ slices, $0 \mathrm{~mm}$ gap, FOV $=256 \mathrm{~mm} \times 256 \mathrm{~mm}$, matrix size $=256 \times 256$, and voxel size $=$ $1291 \mathrm{~mm} \times 1 \mathrm{~mm} \times 1 \mathrm{~mm}$.

\section{Data analysis}

\section{Preprocessing}

132 fMRI data preprocessing was performed with the Data Processing Assistant for Resting133 state fMRI (DPARSF) (Yan \& Zang 2010). To ensure the reliabilty of functional data, we 134 abandoned the first 10 volumes of images. Slice timing and head motion have been done in the remaining 230 volumes of images. No subject had a head movement bigger than $1.5 \mathrm{~mm}$ or rotation bigger than $1.5^{\circ}$. The images were then normalized to the standard echo planar imaging (EPI) template (resampled voxel size: $3 \mathrm{~mm} \times 3 \mathrm{~mm} \times 3 \mathrm{~mm}$ ). The following images were smoothed (full-width at half-maximum Gaussian kernel: $4 \mathrm{~mm}$ ). After normalization, the time series was linearly detrended. Except global signal, 24 parameters of head motion (Friston et al. 1996), signals of white matter, and signals of cerebrospinal fluid were all removed. ALFF/d-

ALFF was based on the frequency spectrum of rs-fMRI signals. work (Ma et al. 2016; Ma et al. 2015).

\section{Static ALFF computation}

ALFF was calculated using DPARSF toolkit as used in prior research (Cheng et al. 2019; Luo et al. 2012). With the aid of fast Fourier transform, the time series was transformed from the

147 time domain to a frequency domain, from which the power spectrum was achieved. With the power spectrum of each voxel from all subjects, the square root was collected at each frequency and then averaged in the region of $0.01-0.08 \mathrm{~Hz}$ (Guo et al. 2013). The square root obtained was known as the ALFF at the given voxel. We divided the ALFF by the global mean ALFF for standardization.

\section{d-ALFF computation}


154

155

156

157

158

159

160

161

162

163

164

165

166

167

168

169

170

171

172

173

174

175

176

177

178

179

180

181

182

183 dependent on DPABI (Yan et al. 2016). According to a former report (Sakoglu et al. 2010), the window length was supposed to be sufficiently short to capture transient signals and long enough to detect slow changing signals. A sliding window with moderate size of $32 \mathrm{TR}$ and a moving step length of 1 TR were selected in this study (Chen et al. 2019b). The 230 time points were divided into 199 windows. ALFF value was computed within each moving window for all participants. Then, the standard deviation (SD) of all ALFF maps from moving windows was computed to evaluate the variability of ALFF. Here, SD was used as d-ALFF.

\section{Statistical analysis}

Statistical analyses were processed with SPM12 toolkit. To compare differences in ALFF and d-ALFF of two groups, we employed two-sample t-tests method. The factors such as age, total GM volume, and gender were regressed. Gaussian Random Field (GRF) approach was adopted to perform multiple comparisons. The voxel level and the cluster level was set $p<0.01$ and $p<0.05$ respectively (the minimum cluster size in ALFF and d-ALFF analyses was 78 voxels) in the GRF correction.

\section{Correlation analysis}

Based on region of interest (ROI), Pearson's correlation was analyzed to probe the relation of alterations in ALFF/d-ALFF to the clinical data of ALS. The mean ALFF/d-ALFF value of each significant clusters (ROIs) was used. A residual term was employed to correlate with clinical data. Meanwhile, the total GM volume, age, and gender were regressed. Bonferroni correction was introduced (significant level: $p<0.05 / N$ ) in the present study. Here, $N=15 / 12$ represented the amount of comparisons using ALFF and d-ALFF.

\section{Classification analysis}

Support vector machine (SVM) method was utilized to compare classification ability among static ALFF, d-ALFF, and their combination for patients/HCs. The mean ALFF and d-ALFF of each static ALFF's ROIs and d-ALFF's ROIs were used as classification features. Liblinear toolbox with default parameter was utilized. Given that we aimed to compare the classification ability among ALFF, d-ALFF, and their combination, a leave-one-out cross validation (LOOCV) was accepted. LOOCV could obtain stable performance and prevent the possibility of overfitting (Chen et al. 2019a; Liu et al. 2015). There were $\mathrm{m}(\mathrm{m}=108)$ LOOCV loops. In each loop, we choose one participants' information to test the categorization model and the $\mathrm{m}-1$ participants' 
184 information was selected for model training. Finally, specificity, sensitivity and accuracy were 185 collected to evaluate classifier performance.

186 Validation analysis

187 In order to confirm the main findings of d-ALFF, d-ALFF data with window lengths of 40 188 TRs and 50 TRs was recollected.

189 Results

190 Differences in static ALFF

191 The ALFF in the ALS group increased in the right inferior temporal gyrus, right medial 192 superior frontal gyrus, and right medial superior frontal gyrus and reduced in the left middle 193 occipital gyrus and left precentral gyrus. The details were available in Table 2 and Figure 1a. 194 Differences in d-ALFF

195 As shown in Table 3 and Figure 1b, d-ALFF did not increase in ALS group. Decreased d196 ALFF was seen in the right lingual gyrus, left superior temporal gyrus, bilateral precentral gyrus, 197 and left paracentral lobule.

198 Correlation analysis

199 No correlation was detected between ALFF and clinical data in ALS. However, as shown in 200 Figure 2, the ALFF in the left superior orbitofrontal gyrus had a negative correlation with 201 ALSFRS-R score at a trend level ( $p=0.0096, \mathrm{r}=-0.3495$, uncorrected). The ALFF in the left 202 superior orbitofrontal gyrus demonstrated a tendency of positive correlation with disease 203 progression rate (Figure 3, $p=0.0135, \mathrm{r}=0.3344$, uncorrected). In addition, no significant 204 difference between d-ALFF and clinical data was found.

\section{Performance of classification}

206 Figure 4 shows the receiver operating characteristic curve (ROC) with ALFF, d-ALFF, and 207 their combination. The numerical data of the area under the curve (AUC) with ALFF, d-ALFF, 208 and their combination were $0.82,0.82$ and 0.84 respectively.

209 As shown in Table 4, the ALFF method showed a classification accuracy of 76.85\%, a 210 specificity of $72.22 \%$, and a sensitivity of $81.48 \%$. The d-ALFF index exhibited a classification 211 accuracy of $76.85 \%$, a specificity of $62.96 \%$, and a sensitivity of $90.74 \%$. The accuracy, 212 specificity, and sensitivity of the combined ALFF and d-ALFF were 79.63\%, 72.22\%, and $21387.04 \%$, respectively.

214 Validation results 
215 The results of d-ALFF using window sizes of 40 TRs and 50 TRs were very similar to the 216 major results of 32 TRs. Validation results were available in Supplementary Materials (Fig. S1a 217 and Fig. S1b).

\section{Discussion}

219 The present research studied the dynamic brain activity in ALS by using d-ALFF for the 220 first time. We found that: (1) patients with ALS showed decreased d-ALFF in the right lingual 221 gyrus, left superior temporal gyrus, bilateral precentral gyrus, and left paracentral lobule at 222 resting state, and (2) the combined ALFF and d-ALFF distinguished ALS from HCs with higher 223 accuracy than ALFF or d-ALFF alone.

\section{Alterations in static ALFF}

225 The brain areas with static ALFF differences in patients with ALS are consistent with 226 previous reports (Luo et al. 2012; Ma et al. 2016), except that the right inferior temporal gyrus 227 had increased ALFF. The temporal lobe with aberrant activation and connection in patients with 228 ALS was discovered in preceding rs-fMRI articles (Li et al. 2018b; Loewe et al. 2017; Zhou et 229 al. 2016). Besides, the thinning of cerebral cortex in the right inferior temporal gyrus is related to rapid clinical progression in ALS (Verstraete et al. 2012). The right inferior temporal gyrus is generally thought to be associated with social information processing for objects, places, and faces (Grill-Spector 2003; Hall et al. 2005). Of note, the cognitive impairment of ALS includes deficits in social cognition and executive functions (Beeldman et al. 2016). Moreover, deficits in recognition of facial expressions of emotion in ALS have been documented (Zimmerman et al. 2007). However, the change in the ALFF value was not found in previous research on ALS (Luo et al. 2012). The finding was probably caused by the situation that patients from the two studies were at different stages of the disease. The previous study recruited patients with an earlier stage compared with that in the present study, where the amplitude feature in this area may not altered. However, more longitudinal research is need to make the result clear. Therefore, this functional alteration may be the imaging evidence for understanding the impaired recognition of emotional stimuli in ALS at a certain stage. Elevated ALFF in the left superior orbitofrontal gyrus was

242 relevant to rate of disease progression and ALSFRS-R score at a trend level. Hence, increased 243 ALFF in this area might be useful to understand the progress of ALS.

\section{Alterations in d-ALFF}


245 Compared with HCs, the d-ALFF in the right lingual gyrus was lower in ALS group.

246 Dysfunction of the right lingual gyrus in ALS was documented, including metabolic difference

247 (Verma et al. 2013) and functional connectivity (Li et al. 2018b). The lingual gyrus is an

248 important area in the visual system (Yang et al. 2015), and 24.13\% of Chinese ALS population

249 are considered with visuospatial disability (Wei et al. 2015). Thus, we concluded that impairment

250 in the right lingual gyrus over time might underlie the phenomenon of visual dysfunction in

251 ALS.

252 This study also found reduced d-ALFF in the left superior temporal gyrus in ALS group.

253 The abnormality in left superior temporal gyrus was same with previous fMRI studies in regional 254 functional connectivity density (Li et al. 2018b) and with anatomical MRI studies in gray matter 255 volume (Buhour et al. 2017; Kim et al. 2017; Sheng et al. 2015). Electroencephalography study 256 (McMackin et al. 2019) shows decreased power in the left superior temporal gyrus when patients 257 with ALS underwent auditory frequency-mismatch oddball paradigm. The left superior temporal 258 gyrus was considered to be related to the function of auditory working memory (Leff et al.

259 2009). The quieter activity in the left superior temporal gyrus over time at resting state in ALS 260 can be explained as the reason of memory decline in ALS.

261 We also observed decreased d-ALFF in the bilateral precentral gyrus and left paracentral 262 lobule in ALS. These motor regions are hallmark areas for patients with ALS who had structural 263 (Cosottini et al. 2012; Schmidt et al. 2014; Thorns et al. 2013) and functional (Ma et al. 2016; 264 Zhang et al. 2017; Zhou et al. 2014) abnormalities. These motor regions were detected with static 265 ALFF and d-ALFF indices in the current research, indicating the vital role of these regions in 266 studying ALS.

\section{Relationship between static ALFF and d-ALFF changes}

$268 \mathrm{~d}$-ALFF and ALFF detected decreased activity in the precentral gyrus in patients suffering 269 from ALS. These findings provide a helpful perspective for our understanding the motor neuron 270 dysfunction of this disease. In addition, d-ALFF could provide other different changes compared 271 with traditional ALFF method, showing that dynamic brain activity may be an important 272 neuroimaging feature to track pathological changes in ALS.

273 Altered d-ALFF could identify patients with ALS from HCs, and the classification 274 performance is similar to that of ALFF. However, when both static and dynamic ALFF features 275 were combined, the classification performance achieved the highest overall accuracy rate. These 
276 results consolidated that ALFF and d-ALFF were different approaches used to characterize brain

277 activity from different perspectives. In contrast to ALFF, d-ALFF could provide complementary

278 information to understand ALS better. The findings also provided a novel way to help

279 distinguish patients with ALS from the healthy population.

\section{Limitations and further considerations}

281 Several limitations should be noted in this work. First, the features in classification were 282 based on prior knowledge, which may increase the overall accuracy rate. The combined ALFF 283 and d-ALFF approach would enhance accuracy with a single feature. More subjects and further 284 sub-group analysis should be considered to obtain stable and more precise results.

\section{Conclusions}

286 ALFF and d-ALFF patterns were altered in patients with ALS. The alterations in the two features could identify ALS at the individual level with nearly the same performance. However, when the two features were combined, the classification performance achieved the highest overall accuracy rate. These results provide evidence for applying dynamic spontaneous neural activity (d-ALFF) to uncover the neuropathology of ALS.

291

292

293

294

295

296

297

298

299

300

301

302

303

304

305

306

307

308

309

310

\section{Acknowledgments}

The research was supported by the Cangzhou Science and Technology Research and Development project (No. 162302137) and China Postdoctoral Science Foundation Grant (No. 2019M653383).

\section{References}

Abidi M, de Marco G, Couillandre A, Feron M, Mseddi E, Termoz N, Querin G, Pradat PF, and Bede P. 2020. Adaptive functional reorganization in amyotrophic lateral sclerosis: coexisting degenerative and compensatory changes. European Journal of Neurology 27:121-128.

Allen EA, Damaraju E, Plis SM, Erhardt EB, Eichele T, and Calhoun VD. 2014. Tracking wholebrain connectivity dynamics in the resting state. Cereb Cortex 24:663-676.

Beeldman E, Raaphorst J, Klein Twennaar M, de Visser M, Schmand BA, and de Haan RJ. 2016. The cognitive profile of ALS: a systematic review and meta-analysis update. J Neurol Neurosurg Psychiatry 87:611-619. 10.1136/jnnp-2015-310734

Biswal B, Zerrin Yetkin F, Haughton VM, and Hyde JS. 1995. Functional connectivity in the motor cortex of resting human brain using echo - planar MRI. Magnetic resonance in medicine 34:537-541.

Bourke SC, Tomlinson M, Williams TL, Bullock RE, Shaw PJ, and Gibson GJ. 2006. Effects of non-invasive ventilation on survival and quality of life in patients with amyotrophic lateral sclerosis: a randomised controlled trial. The Lancet Neurology 5:140-147. https://doi.org/10.1016/S1474-4422(05)70326-4 
311

312

313

314

315

316

317

318

319

320

321

322

323

324

325

326

327

328

329

330

331

332

333

334

335

336

337

338

339

340

341

342

343

344

345

346

347

348

349

350

351

352

353

354

355

356

357

358

359

360

361

Brooks B, Miller RG, Swash M, and Munsat TL. 2000. World Federation of Neurology Research Group on Motor Neuron Diseases. El Escorial revisited: Revised criteria for the diagnosis of amyotrophic lateral sclerosis.

Bueno APA, Pinaya WHL, Rebello K, de Souza LC, Hornberger M, and Sato JR. 2019. Regional dynamics of the resting brain in amyotrophic lateral sclerosis using fractional amplitude of lowfrequency fluctuations and regional homogeneity analyses. Brain connectivity 9:356-364. Buhour M-S, Doidy F, Laisney M, Pitel AL, de La Sayette V, Viader F, Eustache F, and Desgranges B. 2017. Pathophysiology of the behavioral variant of frontotemporal lobar degeneration: A study combining MRI and FDG-PET. Brain imaging and behavior 11:240-252. 10.1007/s11682-016-9521-x

Chen H, Uddin LQ, Guo X, Wang J, Wang R, Wang X, Duan X, and Chen H. 2019a. Parsing brain structural heterogeneity in males with autism spectrum disorder reveals distinct clinical subtypes. Hum Brain Mapp 40:628-637. 10.1002/hbm.24400

Chen J, Sun D, Shi Y, Jin W, Wang Y, Xi Q, and Ren C. 2019b. Dynamic Alterations in Spontaneous Neural Activity in Multiple Brain Networks in Subacute Stroke Patients: A RestingState fMRI Study. Frontiers in neuroscience 12:994-994. 10.3389/fnins.2018.00994

Chen L, Zhang B, Chen R, Tang L, Liu R, Yang Y, Yang Y, Liu X, Ye S, Zhan S, and Fan D. 2015. Natural history and clinical features of sporadic amyotrophic lateral sclerosis in China. Journal of Neurology, Neurosurgery, and Psychiatry 86. 10.1136/jnnp-2015-310471 Cheng C, Dong D, Jiang Y, Ming Q, Zhong X, Sun X, Xiong G, Gao Y, and Yao S. 2019. StateRelated Alterations of Spontaneous Neural Activity in Current and Remitted Depression Revealed by Resting-State fMRI. Front Psychol 10. 10.3389/fpsyg.2019.00245 Chipika RH, Finegan E, Shing SLH, Hardiman O, and Bede P. 2019. Tracking a fast-moving disease: longitudinal markers, monitoring, and clinical trial endpoints in ALS. Frontiers in neurology 10.

Cosottini M, Pesaresi I, Piazza S, Diciotti S, Cecchi P, Fabbri S, Carlesi C, Mascalchi M, and Siciliano G. 2012. Structural and functional evaluation of cortical motor areas in Amyotrophic Lateral Sclerosis. Experimental Neurology 234:169-180. https://doi.org/10.1016/j.expneurol.2011.12.024

Cui Q, Sheng W, Chen Y, Pang Y, Lu F, Tang Q, Han S, Shen Q, Wang Y, and Xie A. 2019. Dynamic changes of amplitude of low - frequency fluctuations in patients with generalized anxiety disorder. Human brain mapping.

Ellis C, Simmons A, Jones D, Bland J, Dawson J, Horsfield M, Williams S, and Leigh P. 1999. Diffusion tensor MRI assesses corticospinal tract damages in ALS.

Friston KJ, Williams S, Howard R, Frackowiak RS, and Turner R. 1996. Movement-related effects in fMRI time-series. Magn Reson Med 35:346-355.

Fu Z, Tu Y, Di X, Biswal BB, Calhoun VD, and Zhang Z. 2017. Associations between functional connectivity dynamics and BOLD dynamics are heterogeneous across brain networks. Frontiers in human neuroscience 11:593.

Grill-Spector K. 2003. The neural basis of object perception. Curr Opin Neurobiol 13:159-166. Guo W-b, Liu F, Xue Z-m, Xu X-j, Wu R-r, Ma C-q, Wooderson SC, Tan C-1, Sun X-1, and Chen J-d. 2012. Alterations of the amplitude of low-frequency fluctuations in treatment-resistant and treatment-response depression: a resting-state fMRI study. Progress in Neuro-

Psychopharmacology and Biological Psychiatry 37:153-160.

Guo W, Liu F, Dai Y, Jiang M, Zhang J, Yu L, Long L, Chen H, Gao Q, and Xiao C. 2013. Decreased interhemispheric resting-state functional connectivity in first-episode, drug-naive major depressive disorder. Progress in Neuro-Psychopharmacology and Biological Psychiatry 41:24-29.

Hall DA, Fussell C, and Summerfield AQ. 2005. Reading fluent speech from talking faces: typical brain networks and individual differences. J Cogn Neurosci 17:939-953.

$10.1162 / 0898929054021175$

Peer] reviewing PDF | (2020:06:49825:1:3:NEW 20 Aug 2020) 
362

363

364

365

366

367

368

369

370

371

372

373

374

375

376

377

378

379

380

381

382

383

384

385

386

387

388

389

390

391

392

393

394

395

396

397

398

399

400

401

402

403

404

405

406

407

408

409

410

411
Hardiman O, Al-Chalabi A, Chio A, Corr EM, Logroscino G, Robberecht W, Shaw PJ, Simmons Z, and van den Berg LH. 2017. Amyotrophic lateral sclerosis. Nature Reviews Disease Primers 3:17071. 10.1038/nrdp.2017.71

Huynh W, Simon NG, Grosskreutz J, Turner MR, Vucic S, and Kiernan MC. 2016. Assessment of the upper motor neuron in amyotrophic lateral sclerosis. Clinical Neurophysiology 127:26432660. https://doi.org/10.1016/j.clinph.2016.04.025

Kawagoe T, Onoda K, and Yamaguchi S. 2019. Subjective memory complaints are associated with altered resting-state functional connectivity but not structural atrophy. NeuroImage: Clinical 21:101675. https://doi.org/10.1016/j.nicl.2019.101675

Kim H-J, de Leon M, Wang X, Kim HY, Lee Y-J, Kim Y-H, and Kim SH. 2017. Relationship between Clinical Parameters and Brain Structure in Sporadic Amyotrophic Lateral Sclerosis Patients According to Onset Type: A Voxel-Based Morphometric Study. PLOS ONE 12:e0168424. 10.1371/journal.pone.0168424

Leff AP, Price CJ, Seghier ML, Schofield TM, Grogan A, Crinion JT, and Green DW. 2009. The left superior temporal gyrus is a shared substrate for auditory short-term memory and speech comprehension: evidence from 210 patients with stroke. Brain 132:3401-3410.

10.1093/brain/awp273

Li J, Duan X, Cui Q, Chen H, and Liao W. 2018a. More than just statics: temporal dynamics of intrinsic brain activity predicts the suicidal ideation in depressed patients. Psychological Medicine:1-9. 10.1017/S0033291718001502

Li W, Zhang J, Zhou C, Hou W, Hu J, Feng H, and Zheng X. 2018b. Abnormal Functional Connectivity Density in Amyotrophic Lateral Sclerosis. Front Aging Neurosci 10:215. 10.3389/fnagi.2018.00215

Liao W, Li J, Ji G-J, Wu G-R, Long Z, Xu Q, Duan X, Cui Q, Biswal BB, and Chen H. 2019. Endless fluctuations: temporal dynamics of the amplitude of low frequency fluctuations. IEEE transactions on medical imaging 38:2523-2532.

Liu F, Guo W, Fouche J-P, Wang Y, Wang W, Ding J, Zeng L, Qiu C, Gong Q, Zhang W, and Chen H. 2015. Multivariate classification of social anxiety disorder using whole brain functional connectivity. Brain Structure and Function 220:101-115. 10.1007/s00429-013-0641-4

Liu F, Guo W, Liu L, Long Z, Ma C, Xue Z, Wang Y, Li J, Hu M, and Zhang J. 2013. Abnormal amplitude low-frequency oscillations in medication-naive, first-episode patients with major depressive disorder: a resting-state fMRI study. Journal of affective disorders 146:401-406. Liu F, Wang Y, Li M, Wang W, Li R, Zhang Z, Lu G, and Chen H. 2017. Dynamic functional network connectivity in idiopathic generalized epilepsy with generalized tonic-clonic seizure. Human brain mapping 38:957-973. 10.1002/hbm.23430

Loewe K, Machts J, Kaufmann J, Petri S, Heinze HJ, Borgelt C, Harris JA, Vielhaber S, and Schoenfeld MA. 2017. Widespread temporo-occipital lobe dysfunction in amyotrophic lateral sclerosis. Sci Rep 7:40252. 10.1038/srep40252

Luo C, Chen Q, Huang R, Chen X, Chen K, Huang X, Tang H, Gong Q, and Shang H. 2012. Patterns of Spontaneous Brain Activity in Amyotrophic Lateral Sclerosis: A Resting-State fMRI Study.

Ma X, Zhang J, Zhang Y, Chen H, Li R, Long Z, Zheng J, Wang J, and Chen H. 2016. Frequency-specific alterations in the fractional amplitude of low-frequency fluctuations in amyotrophic lateral sclerosis. Neurological Sciences:1-9.

Ma X, Zhang J, Zhang Y, Chen H, Li R, Wang J, and Chen H. 2015. Altered cortical hubs in functional brain networks in amyotrophic lateral sclerosis. Neurological Sciences 36:2097-2104. 10.1007/s10072-015-2319-6

McMackin R, Dukic S, Broderick M, Iyer PM, Pinto-Grau M, Mohr K, Chipika R, Coffey A, Buxo T, Schuster C, Gavin B, Heverin M, Bede P, Pender N, Lalor EC, Muthuraman M, Hardiman O, and Nasseroleslami B. 2019. Dysfunction of attention switching networks in 
412

413

414

415

416

417

418

419

420

421

422

423

424

425

426

427

428

429

430

431

432

433

434

435

436

437

438

439

440

441

442

443

444

445

446

447

448

449

450

451

452

453

454

455

456

457

458

459

460

461

amyotrophic lateral sclerosis. NeuroImage: Clinical 22:101707.

https://doi.org/10.1016/j.nicl.2019.101707

Oskarsson B, Gendron TF, and Staff NP. 2018. Amyotrophic Lateral Sclerosis: An Update for

2018. Mayo Clinic Proceedings 93:1617-1628. https://doi.org/10.1016/j.mayocp.2018.04.007

Robberecht W, and Philips T. 2013. The changing scene of amyotrophic lateral sclerosis. Nature

Reviews Neuroscience 14:248-264. 10.1038/nrn3430

Roth KA, and Shacka JJ. 2009. Apoptosis in Neurodegenerative Disease. In: Squire LR, ed.

Encyclopedia of Neuroscience. Oxford: Academic Press, 531-537.

Sakoglu U, Pearlson GD, Kiehl KA, Wang YM, Michael AM, and Calhoun VD. 2010. A method for evaluating dynamic functional network connectivity and task-modulation: application to schizophrenia. MAGMA 23:351-366. 10.1007/s10334-010-0197-8

Schmidt R, Verstraete E, de Reus MA, Veldink JH, van den Berg LH, and van den Heuvel MP. 2014. Correlation between structural and functional connectivity impairment in amyotrophic lateral sclerosis. Human Brain Mapping 35:4386-4395. doi:10.1002/hbm.22481

Shen DC, Hou B, Cui B, Li XL, Peng P, Tai HF, Zhang K, Liu SW, Fu HH, Liu MS, Feng F, and Cui LY. 2018. [Resting-state functional MRI studies of amyotrophic lateral sclerosis patients with various levels of cognitive impairment]. Zhonghua Yi Xue Za Zhi 98:2002-2006.

10.3760/cma.j.issn.0376-2491.2018.25.007

Sheng L, Ma H, Zhong J, Shang H, Shi H, and Pan P. 2015. Motor and extra-motor gray matter atrophy in amyotrophic lateral sclerosis: quantitative meta-analyses of voxel-based morphometry studies. Neurobiology of Aging 36:3288-3299.

https://doi.org/10.1016/j.neurobiolaging.2015.08.018

Sun Y, Dai Z, Li Y, Sheng C, Li H, Wang X, Chen X, He Y, and Han Y. 2016. Subjective cognitive decline: mapping functional and structural brain changes - a combined resting-state functional and structural MR imaging study. Radiology 281:185-192.

Thorns J, Jansma H, Peschel T, Grosskreutz J, Mohammadi B, Dengler R, and Münte TF. 2013. Extent of cortical involvement in amyotrophic lateral sclerosis - an analysis based on cortical thickness. BMC Neurology 13:148. 10.1186/1471-2377-13-148

Turner M, Kiernan MC, Leigh PN, and Talbot K. 2009. Biomarkers in amyotrophic lateral sclerosis. Lancet Neurology 8:94-109.

van Es MA, Hardiman O, Chio A, Al-Chalabi A, Pasterkamp RJ, Veldink JH, and van den Berg LH. 2017. Amyotrophic lateral sclerosis. Lancet 390:2084-2098. 10.1016/S0140-6736(17)312874

Verma G, Woo JH, Chawla S, Wang S, Sheriff S, Elman LB, McCluskey LF, Grossman M, Melhem ER, Maudsley AA, and Poptani H. 2013. Whole-brain analysis of amyotrophic lateral sclerosis by using echo-planar spectroscopic imaging. Radiology 267:851-857.

10.1148/radiol.13121148

Verstraete E, and Foerster BR. 2015. Neuroimaging as a new diagnostic modality in amyotrophic lateral sclerosis. Neurotherapeutics 12:403-416.

Verstraete E, Veldink JH, Hendrikse J, Schelhaas HJ, van den Heuvel MP, and van den Berg LH. 2012. Structural MRI reveals cortical thinning in amyotrophic lateral sclerosis. Journal of Neurology, Neurosurgery \&amp; Psychiatry 83:383-388. 10.1136/jnnp-2011-300909 Volanti P, Cibella F, Sarvà M, De Cicco D, Spanevello A, Mora G, and La Bella V. 2011. Predictors of non-invasive ventilation tolerance in amyotrophic lateral sclerosis. Journal of the neurological sciences 303:114-118.

Wei Q, Chen X, Zheng Z, Huang R, Guo X, Cao B, Bak TH, and Shang H. 2015. Screening for cognitive impairment in a Chinese ALS population. Amyotrophic Lateral Sclerosis and Frontotemporal Degeneration 16:40-45. 10.3109/21678421.2014.966311

Yan C-G, and Zang Y-F. 2010. DPARSF: a MatLab toolbox for "pipeline" data analysis of resting-state fMRI. 
462

463

464

465

466

467

468

469

470

471

472

473

474

475

476

477

478

479

480

481

482

483

484

485

486

487

488

489

490

491

492
Yan CG, Wang XD, Zuo XN, and Zang YF. 2016. DPABI: Data Processing \& Analysis for (Resting-State) Brain Imaging. Neuroinformatics 14:339-351. 10.1007/s12021-016-9299-4 Yang S, Meng Y, Li J, Fan Y-S, Du L, Chen H, and Liao W. 2019. Temporal dynamic changes of intrinsic brain activity in schizophrenia with cigarette smoking. Schizophrenia research 210:6672.

Yang Y-L, Deng H-X, Xing G-Y, Xia X-L, and Li H-F. 2015. Brain functional network connectivity based on a visual task: visual information processing-related brain regions are significantly activated in the task state. Neural regeneration research 10:298-307. 10.4103/16735374.152386

Yu-Feng Z, Yong H, Chao-Zhe Z, Qing-Jiu C, Man-Qiu S, Meng L, Li-Xia T, Tian-Zi J, and YuFeng W. 2007. Altered baseline brain activity in children with ADHD revealed by resting-state functional MRI. Brain and Development 29:83-91.

Zhang C, Dou B, Wang J, Xu K, Zhang H, Sami MU, Hu C, Tao R, Chen N, and Li K. 2019. Dynamic Alterations of Spontaneous Neural Activity in Parkinson's Disease: A Resting-State fMRI Study. Frontiers in neurology 10:1052.

Zhang J, Ji B, Hu J, Zhou C, Li L, Li Z, Huang X, and Hu X. 2017. Aberrant interhemispheric homotopic functional and structural connectivity in amyotrophic lateral sclerosis. $J$ Neurol Neurosurg Psychiatry 88:369-370. 10.1136/jnnp-2016-314567

Zhou C, Hu X, Hu J, Liang M, Yin X, Chen L, Zhang J, and Wang J. 2016. Altered Brain Network in Amyotrophic Lateral Sclerosis: A Resting Graph Theory-Based Network Study at Voxel-Wise Level. Frontiers in neuroscience 10:204-204. 10.3389/fnins.2016.00204

Zhou F, Xu R, Dowd E, Zang Y, Gong H, and Wang Z. 2014. Alterations in regional functional coherence within the sensory-motor network in amyotrophic lateral sclerosis. Neuroscience Letters 558:192-196. https://doi.org/10.1016/j.neulet.2013.11.022

Zimmerman EK, Eslinger PJ, Simmons Z, and Barrett AM. 2007. Emotional perception deficits in amyotrophic lateral sclerosis. Cogn Behav Neurol 20:79-82. 10.1097/WNN.0b013e31804c700b Zou Q, Wu CW, Stein EA, Zang Y, and Yang Y. 2009. Static and dynamic characteristics of cerebral blood flow during the resting state. Neuroimage 48:515-524.

https://doi.org/10.1016/j.neuroimage.2009.07.006 


\section{Figure 1}

Results of ALFF and d-ALFF analyses by two-sample t-tests between ALS group and HC group.

a. Brain regions with significant difference in static ALFF between the ALS group and HC group. b. Brain regions with significant difference in d-ALFF between the ALS group and HC group. The voxel level was set at $p<0.01$, and the cluster level was set at $p<0.05$ with GRF corrected. The color bar represents the T value of the between-group analysis. Hot colors represent higher ALFF/d-ALFF in the ALS group than in the healthy control group, and cool colors represent the lower ALFF/d-ALFF in the ALS group than the healthy control group.

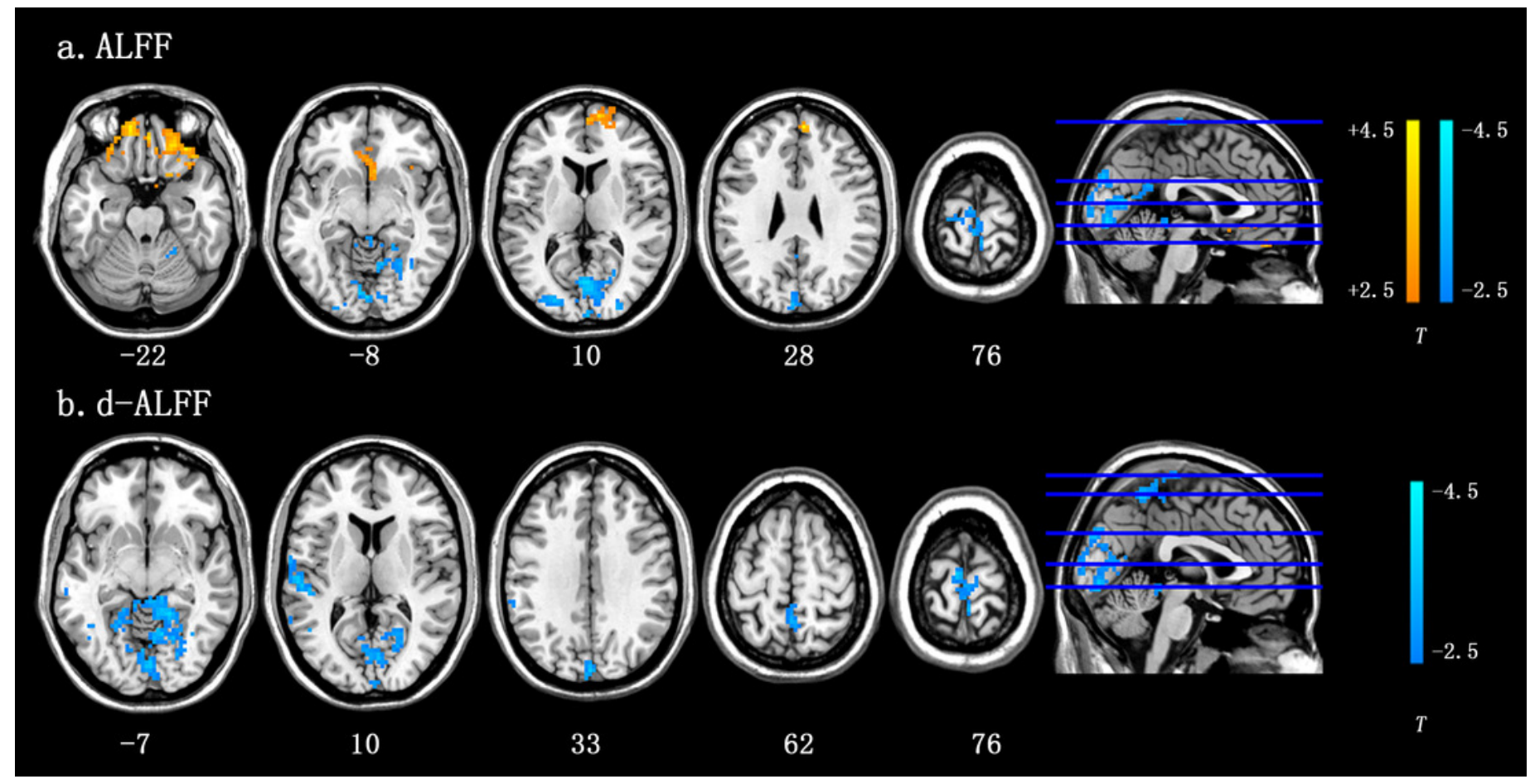


Figure 2

Correlation between ALFF value in the left superior orbitofrontal gyrus and ALSFRS-R score in the ALS group.

Correlation between ALFF value in the left superior orbitofrontal gyrus and ALSFRS-R score in the ALS group.

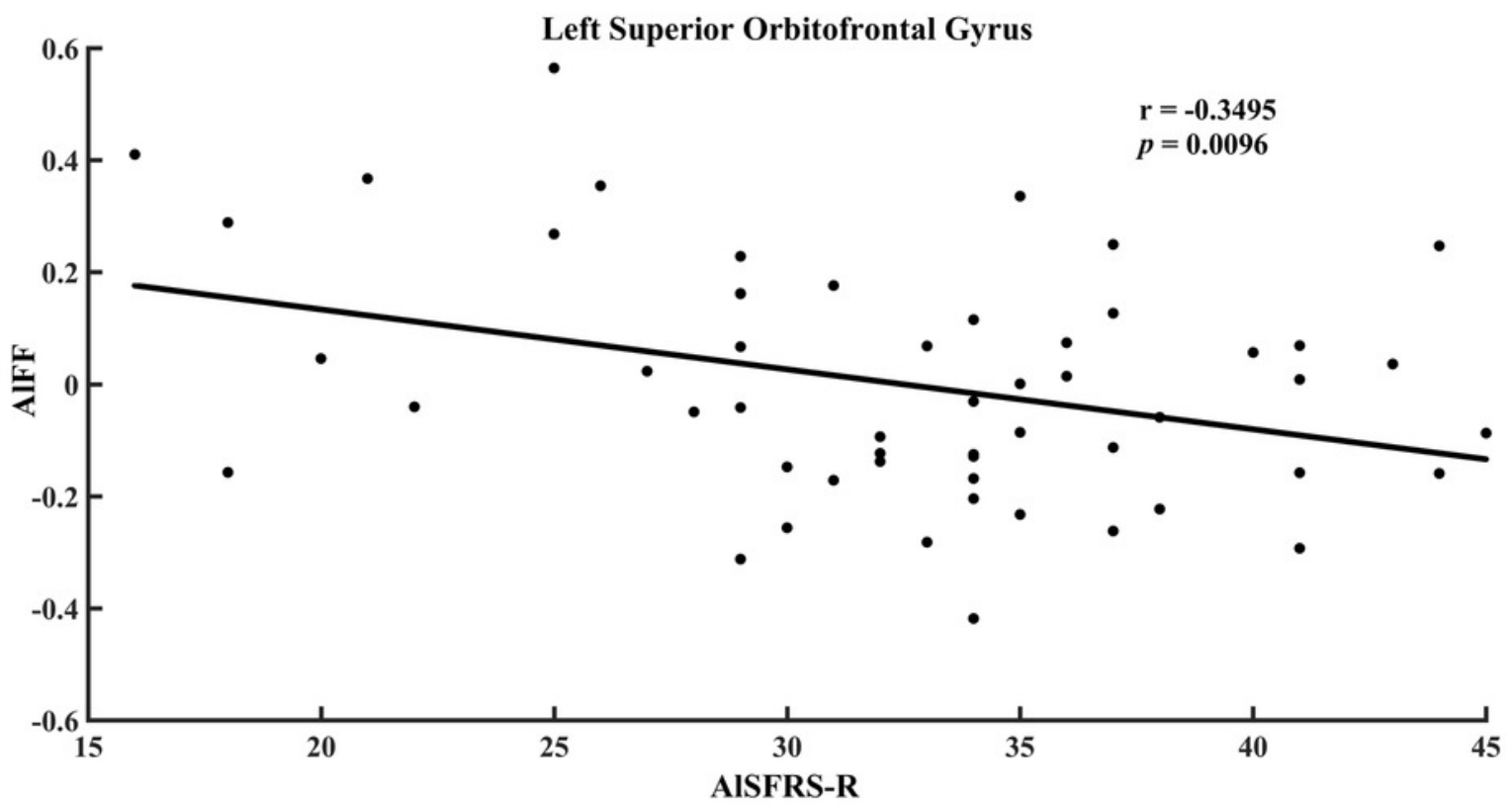


Figure 3

Correlation between ALFF value in the left superior orbitofrontal gyrus and disease progression rate in the ALS group.

Correlation between ALFF value in the left superior orbitofrontal gyrus and disease progression rate in the ALS group.

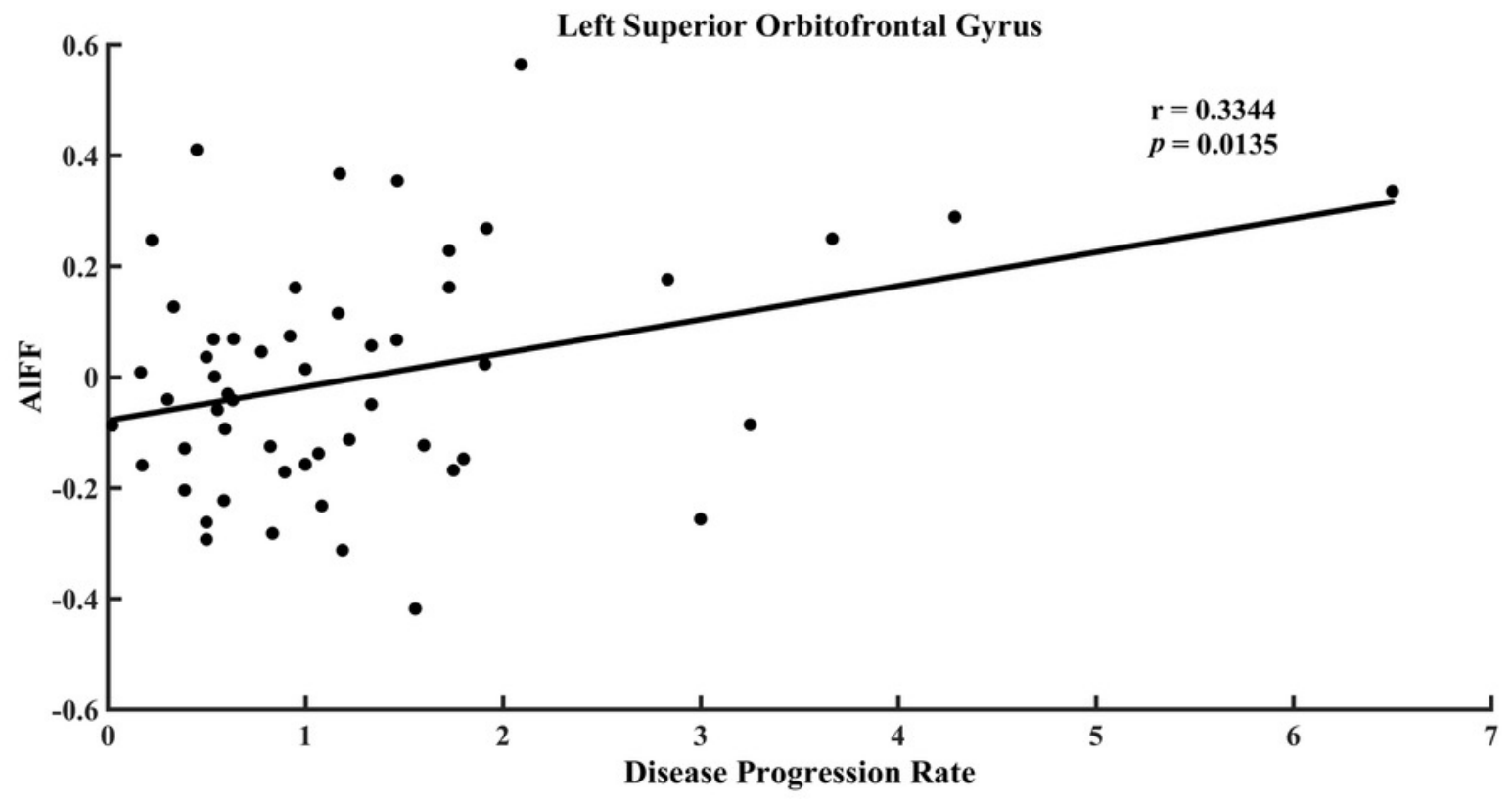


Figure 4

Receiver operating characteristic curve of the classifier with ALFF, d-ALFF, and their combination.

Receiver operating characteristic curve of the classifier with ALFF, d-ALFF, and their combination.

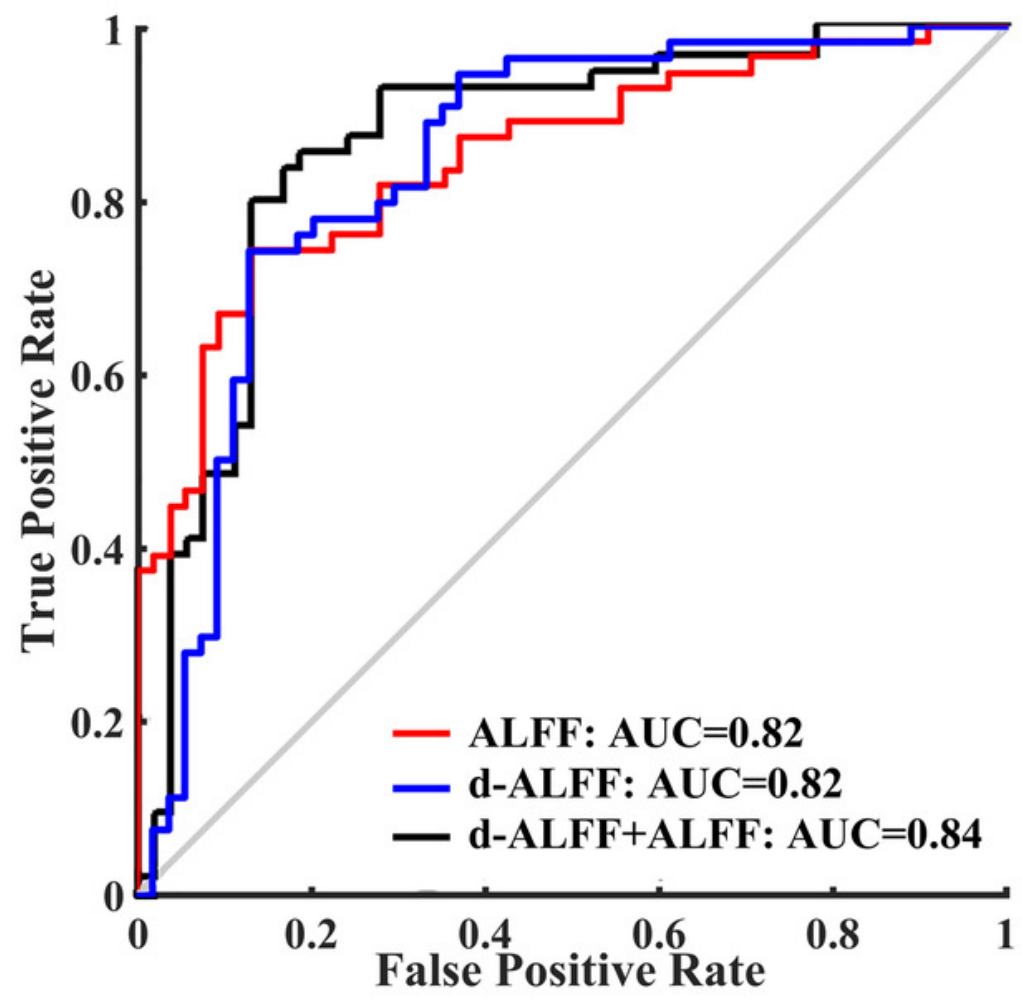




\section{Table $\mathbf{1}$ (on next page)}

Demographic and clinical characteristics of the ALS patients and HCs.

Values are mean \pm variance. ALS, Amyotrophic Lateral Sclerosis. HC, Healthy Control. ALSFRS-R, ALS Functional Rating Scale-revised. Disease progression rate, (48-ALSFRS-R

score) / time from symptom onset. ${ }^{\mathrm{a}}$ The $p$ value was obtained by Chi-square t-test. ${ }^{\mathrm{b}}$ The $p$ value was obtained by two-sample t-test. 
Table 1. Demographic and clinical characteristics of the ALS patients and HCs.

\begin{tabular}{llll}
\hline Variables & ALS (n = 54) & HC (n= 54) & $p$ value \\
\hline Gender (female/male) & $18 / 36$ & $18 / 36$ & $1^{\mathrm{a}}$ \\
Age (years) & $48.71 \pm 10.21$ & $48.30 \pm 8.74$ & $0.82^{\mathrm{b}}$ \\
Gray matter & $649.56 \pm 2842.17$ & $668.23 \pm 3251.82$ & $0.08^{\mathrm{b}}$ \\
Disease duration (months) & $20.93 \pm 21.56$ & - & - \\
ALSFRS-R & $32.56 \pm 6.83$ & - & - \\
Disease progression rate & $1.28 \pm 1.15$ & - & - \\
\hline
\end{tabular}

Values are mean \pm variance. ALS, Amyotrophic Lateral Sclerosis. HC, Healthy Control. ALSFRS-R, ALS Functional Rating Scale-revised. Disease progression rate, (48-ALSFRS-R score) / time from symptom onset. ${ }^{\text {a }}$ The $p$ value was obtained by Chi-square t-test. ${ }^{\mathrm{b}}$ The $p$ value was obtained by twosample t-test. 


\section{Table 2 (on next page)}

Differences in ALFF between ALS and HC groups.

MNI, Montreal Neurological Institute. $X, Y, Z$, coordinates of primary peak locations in the MNI space. ALS, Amyotrophic Lateral Sclerosis. HC, Healthy Control. T value denotes the statistic value of two-sample t-test by contrasting the ALS patients to the controls ( $p<0.01$, GRFcorrected at a cluster level of $p<0.05$ ). 
1 Table 2. Differences in ALFF between ALS and HC groups.

\begin{tabular}{|c|c|c|c|c|}
\hline \multirow{3}{*}{ Clusters } & \multirow{3}{*}{ Brain regions } & Cluster size & MNI & \multirow{3}{*}{$\mathrm{T}$ value } \\
\hline & & & & \\
\hline & & (voxels) & $(\mathrm{X}, \mathrm{Y}, \mathrm{Z})$ & \\
\hline \multicolumn{5}{|c|}{$\mathbf{A L S}>\mathbf{H C}$} \\
\hline Cluster 1 & Right inferior temporal gyrus & 128 & $45,-6,-45$ & 4.14 \\
\hline Cluster 2 & Left superior orbitofrontal gyrus & 442 & $-15,18,-15$ & 4.45 \\
\hline Cluster 3 & Right medial superior frontal gyrus & 93 & $6,54,27$ & 4.35 \\
\hline \multicolumn{5}{|c|}{$\mathbf{A L S}<\mathbf{H C}$} \\
\hline Cluster 1 & Left middle occipital gyrus & 895 & $-27,90,-3$ & -4.56 \\
\hline Cluster 2 & Left precentral gyrus & 108 & $-27,-21,72$ & -3.81 \\
\hline
\end{tabular}

2 MNI, Montreal Neurological Institute. X, Y, Z, coordinates of primary peak locations in the MNI space. ALS,

3 Amyotrophic Lateral Sclerosis. HC, Healthy Control. T value denotes the statistic value of two-sample t-test by

4 contrasting the ALS patients to the controls $(p<0.01$, GRF-corrected at a cluster level of $p<0.05)$. 


\section{Table 3 (on next page)}

Differences in d-ALFF between ALS and HC groups.

MNI, Montreal Neurological Institute. $X, Y, Z$, coordinates of primary peak locations in the MNI space. ALS, Amyotrophic Lateral Sclerosis. HC, Healthy Control. T value denotes the statistic value of two-sample t-test by contrasting the ALS patients to the controls ( $p<0.01$, GRFcorrected at a cluster level of $p<0.05$ ). 
1 Table 3. Differences in d-ALFF between ALS and HC groups.

\begin{tabular}{|c|c|c|c|c|}
\hline \multirow{3}{*}{ Clusters } & \multirow{3}{*}{ Brain regions } & Cluster size & & $\mathrm{T}$ \\
\hline & & \multirow{2}{*}{\multicolumn{2}{|c|}{ MNI $(\mathrm{X}, \mathrm{Y}, \mathrm{Z})$}} & \\
\hline & & & & value \\
\hline \multicolumn{5}{|c|}{$\mathbf{A L S}>\mathbf{H C}$} \\
\hline \multicolumn{5}{|l|}{ None } \\
\hline \multicolumn{5}{|c|}{$\mathbf{A L S}<\mathbf{H C}$} \\
\hline Cluster 1 & Right lingual gyrus & 988 & $24,-45,-9$ & -4.35 \\
\hline Cluster 2 & Left superior temporal gyrus & 176 & $-48,-27,12$ & -3.82 \\
\hline Cluster 3 & Bilateral precentral gyrus & 90 & $51,-9,24$ & -3.45 \\
\hline Cluster 4 & Left paracentral lobule & 125 & $-6,-15,75$ & -3.70 \\
\hline
\end{tabular}

2 MNI, Montreal Neurological Institute. X, Y, Z, coordinates of primary peak locations in the MNI space. ALS,

3 Amyotrophic Lateral Sclerosis. HC, Healthy Control. T value denotes the statistic value of two-sample t-test by

4 contrasting the ALS patients to the controls $(p<0.01$, GRF-corrected at a cluster level of $p<0.05)$. 


\section{Table 4 (on next page)}

Performance evaluation of classifier using ALFF, d-ALFF and combined ALFF and d-ALFF. 
1 Table 4. Performance evaluation of classifier using ALFF, d-ALFF and combined ALFF and d-ALFF.

\begin{tabular}{llll} 
& Sensitivity (\%) & Specificity (\%) & Accuracy (\%) \\
\hline ALFF & 81.48 & 72.22 & 76.85 \\
d-ALFF & 90.74 & 62.96 & 76.85 \\
combined ALFF and d-ALFF & 87.04 & 72.22 & 79.63 \\
\hline
\end{tabular}

2

3 